\title{
On Pontrjagin classes and homotopy types of manifolds.
}

\author{
By Itiro TAMURA
}

(Received March 15, 1957)

\section{Introduction.}

In the present paper we shall obtain manifolds of the same homotopy type with different Pontrjagin classes, belonging therefore to different classes in the sense of diffeomorphism (i. e. differentiable homeomorphism).

This reveals the Pontrjagin classes as no homotopy invariants and the problem of "topological invariance of Pontrjagin classes," except for mod 2 and mod 3, as not provable by means of homotopy invariants, such as (co)homology groups, homotopy groups, Steenrod operators etc.

In section 2 of this paper we define some sphere bundles over spheres and determine the homotopy types of them by method of $A$. Dold. In section 4, Pontrjagin classes of these bundles are calculated from the Chern classes of associated bundles by the obstruction theory prepared in section 3. Section 5 is devoted to the description of the cohomology groups of total spaces of bundles. The principal tool here is the Gysin exact sequence.

In section $6, C^{\infty}$-manifolds are defined from the bundles and their Pontrjagin classes are computed. Our final results are exposed in section 7.

We use in this paper the results on the homotopy groups of spheres and classical groups which can be found, for example, in Steenrod [11], Borel et Serre [1], Serre [10].

The author wishes to express his hearty thanks to his friends $\mathrm{T}$. Nakamura, A. Hattori for their kind discussions and valuable suggestions, and also to Professor S. Iyanaga for his constant encouragement. 


\section{Fibre bundles over the $q$-sphere.}

Let $\mathfrak{B}=\left\{B, p, S^{q}, S^{r}, S O(r+1)\right\}$ be fibre bundles over $q$-sphere $S^{q}$ with total space $B, r$-sphere $S^{r}$ as fibre and the rotation group $S O(r+1)$ as structural group.

Let $S^{q-1}$ be a great $(q-1)$-sphere on $S^{q}$ and let $E_{1}, E_{2}$ be the closed hemispheres of $S^{q}$ determined by $S^{q-1}$. Let $V_{i}(i=1,2)$ be an open $q$-cell on $S^{q}$ containing $E_{i}$ and bounded by an $(q-1)$-sphere parallel to $S^{q-1}$, and $x_{0}$ be a reference point on $S^{q-1}$.

Bundle $\mathfrak{B}$ is strictly equivalent to a bundle with coordinate neighbourhoods $V_{i}(i=1,2)$ and coordinate functions $\phi_{i}: V_{i} \times S^{r} \rightarrow \mathfrak{B}_{i}(i=1,2)$, where $\mathfrak{B}_{i}$ is the portion of $\mathfrak{B}$ over $V_{i}$, and with coordinate transformations $g_{12}$ such that $g_{12}\left(x_{0}\right)=e$.

The map $T=g_{12} \mid S^{q-1}$ which maps $S^{q-1}$ into $S O(r+1)$ is characteristic map of $\mathfrak{B}$. The equivalence classes of bundles are in 1-1 correspondence with homotopy classes of maps $T$.

For $q=4,8$, we shall describle the homotopy classes of maps $T$ explicitly.

As is well-known, we have

$$
\begin{array}{ll}
\pi_{3}(S O(3)) \approx Z, \pi_{3}(S O(4)) \approx Z+Z, \pi_{3}(S O(r)) \approx Z & (r \geqq 5), \\
\pi_{7}(S O(7)) \approx Z, \pi_{7}(S O(8)) \approx Z+Z, \pi_{7}(S O(r)) \approx Z & (r \geqq 9) .
\end{array}
$$

( $Z$ means as usual the additive group of integers, $Z_{n}$ the group $Z$ $\bmod n$.)

Let $i_{r}: S O(r) \rightarrow S O(r+1)$ be natural injection. Then the generators

$$
\left\{\rho_{3}\right\},\left\{\rho_{4}, \sigma_{4}\right\},\left\{\sigma_{r}\right\}(r>5),\left\{\overline{\boldsymbol{\rho}}_{7}\right\},\left\{\overline{\boldsymbol{\rho}}_{8}, \overline{\boldsymbol{\sigma}}_{8}\right\},\left\{\overline{\boldsymbol{\sigma}}_{r}\right\} \quad(\boldsymbol{r} \geqq 9)
$$

of $\pi_{3}(S O(3)), \pi_{3}(S O(4)), \pi_{3}(S O(r)), \pi_{7}(S O(7)), \pi_{7}(S O(8)), \pi_{7}(S O(r))$ respectively are given as follows;

$$
\rho_{3}(u) v=u v u^{-1}, \rho_{4}=\left(i_{3}\right)_{*} \rho_{3}, \sigma_{4}(u) v=u v,
$$

where $u, v$ denote quaternions as usual. And

$$
\begin{aligned}
& \sigma_{r}=\left(i_{r-1}\right)_{*} \circ\left(i_{r-2}\right)_{*} \circ \cdots \circ\left(i_{4}\right)_{*} \sigma_{4} \quad(r \geqq 5) . \\
& t \bar{\rho}_{7}(x) y=x y x^{1}, \bar{\rho}_{8}=\left(i_{7}\right)_{*} \bar{\rho}_{7}, \bar{\sigma}_{8}(x) y=x y,
\end{aligned}
$$

where $x, y$ denote Cayley numbers as usual and $t$ is a odd integer. ${ }^{1)}$ Furthermore

$$
\bar{\sigma}_{9}=\left(i_{8}\right)_{*}\left(\bar{\sigma}_{8}-[t / 2] \bar{\rho}_{8}\right),{ }^{2)} \bar{\sigma}_{r}=\left(i_{r-1}\right)_{*} \circ\left(i_{r-2}\right)_{*} \circ \cdots \circ\left(i_{9}\right)_{*} \bar{\sigma}_{9} \quad(r \geqq 10) .
$$

Between these generators hold the following relations. 


$$
\left(i_{4}\right)_{*} \rho_{4}=2 \sigma_{5},\left(i_{8}\right)_{*} \bar{\rho}_{8}=2 \bar{\sigma}_{9},\left(i_{8}\right)_{*} \bar{\sigma}_{8}=t \bar{\sigma}_{9} .
$$

Now we define the bundles $\mathfrak{B}^{(q, r)}$ by:

$$
\begin{array}{ll}
\mathfrak{B}_{m, n}^{(4,3)}=\left\{B_{m, n}^{(4,3)}, p, S^{4}, S^{3}, S O(4)\right\}, & \\
\mathfrak{B}_{n}^{(4, r)}=\left\{B_{n}^{(4, r)}, p, S^{4}, S^{r}, S O(r+1)\right\} & (r \geqq 4), \\
\mathfrak{B}_{m, n}^{(8,7)}=\left\{B_{m, n}^{(8,7)}, p, S^{8}, S^{7}, S O(8)\right\}, & \\
\mathfrak{B}_{n}^{(8, r)}=\left\{B_{n}^{(8, r)}, p, S^{8}, S^{r}, S O(r+1)\right\} & (r \geqq 8),
\end{array}
$$

where $\mathfrak{B}_{m, n}^{(4,3)}, \mathfrak{B}_{n}^{(4, r)}, \mathfrak{B}_{m, n}^{(8,7)}, \mathfrak{B}_{n}^{(8, r)}$ have characteristic maps $m \rho_{4}+\boldsymbol{n} \sigma_{4}, \boldsymbol{n} \sigma_{r}$, $m \bar{\rho}_{8}+n \bar{\sigma}_{8}, n \bar{\sigma}_{r}$ respectively.

Let us consider the commutative diagram;

$$
\begin{gathered}
\pi_{q-1}(S O(r)) \stackrel{\left(i_{r}\right)_{*}}{\longrightarrow} \pi_{q-1}(S O(r+1)) \\
\int_{\pi_{q-1}}^{\downarrow}\left(G^{r}\right) \stackrel{\left(\bar{j}_{0}\right)_{*}}{\stackrel{(i))_{*}}{\longrightarrow}} \pi_{q-1}\left(\mathbb{( S S}^{r}\right)
\end{gathered}
$$

where $\left(S^{r}\right.$ is the space of all continuous mappings from $S^{r}$ into $S^{r}$ with the compact open topology, $G^{r}$ is the subspace of $\mathbb{S}^{r}$ consisting of mappings which leave invariant one fixed point of $S^{r}$ and $\bar{j}_{0}, \bar{j}, \bar{\imath}$ are natural injections.

We owe Dold [2] the following theorem on the homotopy equivalence between sphere bundles over spheres.

THEOREM 2.1. Let $T_{i}$ be the characteristic map of $\mathfrak{B}_{i}=\left\{B_{i}, p, S^{q}\right.$, $\left.S^{r}, S O(r+1)\right\}(i=1,2)$. Let $(\bar{j})_{*}$ be the map of $\pi_{q-1}(S O(r+1))$ into $\pi_{q-1}\left(\mathbb{S}^{r}\right)$ as given by (1.3). If $(\bar{j})_{*} T_{1}=(\bar{j})_{*} T_{2}$, then $T_{1}$ and $T_{2}$ define homotopically equivalent bundles.

As is well known, we have $\iota: \pi_{q-1}\left(G^{r}\right) \approx \pi_{r+q-1}\left(S^{r}\right)$ and $J=\iota 0\left(\bar{j}_{0}\right)_{*}$ : $\pi_{q-1}(S O(r)) \rightarrow \pi_{r+q-1}\left(S^{r}\right)$ is the so-called $J$ homomorphism (G. W. Whitehead [14]). And

$$
\begin{array}{lll}
\pi_{6}\left(S^{3}\right) \approx Z_{12}, \pi_{7}\left(S^{4}\right) \approx Z+Z_{12}, & \pi_{3+r}\left(S^{r}\right) \approx Z_{24} & (r \geqq 5) ; \\
\pi_{14}\left(S^{7}\right) \approx Z_{120}, \pi_{15}\left(S^{8}\right) \approx Z+Z_{120}, \pi_{7+r}\left(S^{r}\right) \approx Z_{240} & (r \geqq 9) .
\end{array}
$$

Therefore, in the following commutative diagram,

we have

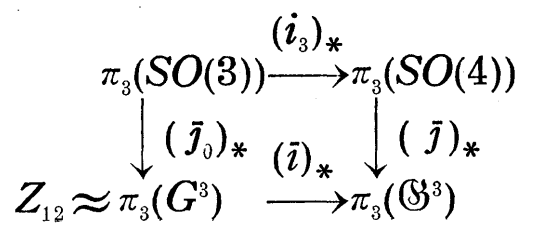




$$
(\bar{\jmath})_{*}\left(12 \rho_{4}\right)=(\bar{\jmath})_{*} \circ\left(i_{3}\right)_{*}\left(12 \rho_{3}\right)=(\bar{l})_{*} \circ\left(\bar{j}_{0}\right)_{*}\left(12 \rho_{3}\right)=0 \text {. }
$$

Similarly we have following commutative diagram,

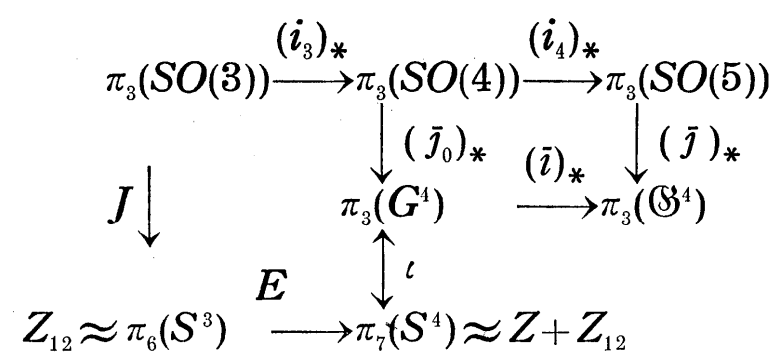

where $E$ is the suspension homomorphism. Therefore

$$
\begin{aligned}
(\bar{\jmath})_{*}\left(24 \sigma_{5}\right) & =(\bar{\jmath})_{*} \circ\left(i_{4}\right)_{*}\left(12 \rho_{4}\right)=(\bar{\imath})_{*} \circ\left(j_{0}\right)_{*}\left(12 \rho_{4}\right) \\
& =(\bar{l})_{*} \circ\left(j_{0}\right)_{*} \circ\left(i_{3}\right)_{*}\left(12 \rho_{3}\right)=(\bar{\imath})_{*} \circ \iota^{-1} \circ E \circ J\left(12 \rho_{3}\right)=0 .
\end{aligned}
$$

For $r \geq 5$, we have the following commutative diagram,

$$
\begin{aligned}
\pi_{3}(S O(r)) \stackrel{\left(i_{r}\right)_{*}}{\longrightarrow} \pi_{3}(S O(r+1)) \\
Z_{24} \approx \pi_{3}\left(G^{r}\right) \stackrel{\left.(\bar{\imath})_{*}\right|^{\downarrow}(\bar{\jmath})_{*}}{\longrightarrow} \pi_{3}\left(\mathbb{S}^{r}\right)
\end{aligned}
$$

and

$$
(\bar{j})_{*}\left(24 \sigma_{r+1}\right)=(\bar{j})_{*} \circ\left(i_{r}\right)_{*}\left(24 \sigma_{r}\right)=(\bar{\imath})_{*} \circ\left(\bar{j}_{0}\right)_{*}\left(24 \sigma_{r}\right)=0 .
$$

By Theorem 2.1 and the above results, we obtain easily the following Theorem using weak equivalence of bundles.

THEOREM 2.2. (i) If $m \equiv m^{\prime}(\bmod 12), n=n^{\prime}$; or $m=-m^{\prime}, n=-n^{\prime}$, then $B_{m, n}^{(4,3)}, B_{m^{\prime}, n^{\prime}}^{(4,3)}$ have the same homotopy type.

(ii) Let $r \geqq 4$. If $n \equiv \pm n^{\prime}(\bmod 24)$, then $B_{n}^{(4, r)}, B_{n^{\prime}}^{(4, r)}$ have the same homotopy type.

Similarly we obtain for $\mathfrak{B}_{m, n}^{(8,7)}$ and $\mathfrak{B}_{n}^{(8, r)}$.

THEOREM 2.3. (i) If $m \equiv m^{\prime}(\bmod 120), n=n^{\prime}$; or $m=-m^{\prime}, n=$ $-n^{\prime}$; then $B_{m, n}^{(8,7)}, B_{m^{\prime}, n^{\prime}}^{(8,7)}$ have the same homotopy type.

(ii) Let $r \geqq 8$, If $n \equiv \pm n^{\prime}(\bmod 240)$, then $B_{n}^{(8, r)}, B_{n^{\prime}}^{(8, r)}$ have the same homotopy type.

REMARK 2.4. James and J.H. C. Whitehead [7] have given a necessary and sufficient condition for sphere bundles over spheres with cross sections to have the same homotopy type. We see by their result that the conditions of Theorem 2.2 are also necessary in case of $B_{m, 0}^{(4,3)}, B_{n}^{(4, q)}$. 


\section{Obstructions of bundles over $q$-sphere.}

Let $\mathfrak{B}^{\prime}=\left\{B^{\prime}, p^{\prime}, S^{q}, Y, S O(r+1)\right\}$ be a bundle (weakly) associated to $\mathfrak{B}$, and we now assume that $\pi_{i}(Y)=0(0 \leqq i<q-1), \pi_{q-1}(Y) \approx Z$. We denote with $\varepsilon$ a generator of $\pi_{q-1}(Y)$.

By our assumptions obstruction cocycle $\bar{c}\left(\mathfrak{B}^{\prime}\right) \in H^{q}\left(S^{q}\right)$ is defined (Steenrod [11] §32). Let us compute $\bar{c}\left(\mathfrak{B}^{\prime}\right)$ from the characteristic map $T$ of $\mathfrak{B}$.

Let $E_{1}, E_{2}$ be hemispheres of $S^{q}$ as in section 2. They constitute a cell subdivision of $S^{q}$. Orient $E_{1}, E_{2}$ concordantly with $S^{q}$ and orient $S^{q-1}$ so as to be positively incident with $E_{1}$.

We construct a cross section of $\mathfrak{B}^{\prime}$ over $E_{2}$ by $\phi_{2}\left(E_{2} \times y_{0}\right)$, where $y_{0}$ is a fixed point of fibre $Y$. This cross section is defined over $S^{q-1}$ and obstruction to extend it over $E_{1}$ is given by $\phi_{1}^{-1} \phi_{2}\left(S^{q-1} \times y_{0}\right)=$ $T(x)\left(y_{0}\right), x \in S^{q-1}$. Define now $[T(x)]$ by

$$
T(x)\left(y_{0}\right) \in[T(x)] \varepsilon .
$$

Then we have $\bar{c}\left(\mathfrak{B}^{\prime}\right)\left(E_{1}\right)=[T(x)]$ and $\bar{c}\left(\mathfrak{B}^{\prime}\right)\left(E_{2}\right)=0$. Hence we obtain the following theorem:

THEOREM 3.1. Under above hypothesis, the primary obstruction cocycle $\bar{c}\left(\mathfrak{B}^{\prime}\right)$ of $\mathfrak{B}^{\prime}$ is given by $\pm[T(x)] \alpha$, where $\alpha$ is a generator of $H^{q}\left(S^{q}\right)$.

\section{Pontrjagin classes of fibre bundles.}

Pontragin classes of a fibre bundle $\mathfrak{B}$ with structural group $S O(r)$ are defined as follows (Hirzebruch [5] § 4).

Let $j_{r}: S O(r) \rightarrow S U(r)$ be natural injection of the rotation group into the special unitary group. We can regard bundle $\mathfrak{B}$ provided with structural group $S U(r)$.

We construct associate bundles $\mathfrak{B}_{i}^{\prime}(i=1,2, \cdots, r)$ with fibre $S U(r) / S U(i-1)$. Then Pontrjagin classes $p_{i}(i=1,2, \cdots,[r / 2])$ of $\mathfrak{B}$ are defined by

$$
p_{i}(\mathfrak{B})=(-1)^{i} \bar{c}\left(\mathfrak{B}_{2 i}^{\prime}\right) .
$$

Let us calculate $p_{1}$ of $\mathfrak{B}^{(4, r)}(r \geqq 3)$ and $p_{2}$ of $\mathfrak{B}^{(8, r)}(r \geqq 7)$. We denote $\mathfrak{B}_{2}^{\prime(4, r)}, \mathfrak{B}_{4}^{\prime(8, r)}$ simply by $\mathfrak{B}^{(4, r)}, \mathfrak{B}^{\prime(8, r)}$.

(I) The first Pontrjagin class of $\mathfrak{B}^{(4, r)}(r \geqq 3)$.

Since natural projection $p: S U(r) \rightarrow S U(r) / S U(1)$ induces the isomorphism of homotopy groups 


$$
p_{*}: \pi_{3}(S U(r)) \approx \pi_{3}(S U(r) / S U(1)) \quad(r \geqq 3),
$$

we may consider $\pi_{3}(S U(r))$, instead of $\pi_{3}(S U(r) / S U(1))$.

Let us consider the following (not commutative) diagram, where $i_{r}^{\prime}, k_{r}, j_{r}^{\prime}$ are natural injections and $d: S O(r) \rightarrow S O(r) \times S O(r)$ is defined by $a \rightarrow(a, a)$.




We have $\pi_{3}(\mathrm{~S} U(r)) \approx Z(r \geqq 2)$. Let $\mu_{r}$ be a generator of $\pi_{3}(S U(r))$ such that

$$
\left(i_{r}^{\prime}\right)_{*} \mu_{r}=\mu_{r+1}, \quad\left(k_{2}\right)_{*} \mu_{2}=\sigma_{4}
$$

Then we have

$$
\begin{aligned}
\left(k_{4}\right)_{*} \circ\left(j_{4}\right)_{*}\left(m \rho_{4}+n \sigma_{4}\right) & =\left(j_{4}^{\prime}\right)_{*} \circ(d)_{*}\left(m \rho_{4}+n \sigma_{4}\right) \\
& =2\left(i_{7}\right)_{*} \circ\left(i_{6}\right)_{*} \circ\left(i_{5}\right)_{*} \circ\left(i_{4}\right)_{*}\left(m \rho_{4}+n \sigma_{4}\right) .
\end{aligned}
$$

Since $\left(i_{r}\right)_{*}$ is isomorphism onto for $r \geqq 5$ and

$$
\left(i_{4}\right)_{*} \sigma_{4}=\sigma_{5},\left(i_{4}\right)_{*} \rho_{4}=2 \sigma_{5}
$$

we have

$$
\left(k_{4}\right)_{*} \circ\left(j_{4}\right)_{*}\left(m \rho_{4}+n \sigma_{4}\right)=2(2 m+n) \sigma_{8} \cdot
$$

On the other hand

$$
\begin{aligned}
\mu_{4}= & \left(i_{3}^{\prime}\right)_{*} \circ\left(i_{2}^{\prime}\right)_{*} \mu_{2}, \sigma_{8}=\left(i_{7}\right)_{*} \circ\left(i_{6}\right)_{*} \circ\left(i_{5}\right)_{*} \circ\left(i_{4}\right)_{*} \circ\left(k_{2}\right)_{*} \mu_{2}, \\
& \left(k_{4}\right)_{*} \circ\left(i_{3}^{\prime}\right)_{*} \circ\left(i_{2}^{\prime}\right)_{*}=\left(i_{7}\right)_{*} \circ\left(i_{6}\right)_{*} \circ\left(i_{5}\right)_{*} \circ\left(i_{4}\right)_{*} \circ\left(k_{2}\right)_{*} .
\end{aligned}
$$

Therefore

$$
\mu_{4}=\left(k_{4}\right)_{*}^{-1} \sigma_{8}
$$

and

$$
\left(j_{4}\right)_{*}\left(m \rho_{4}+n \sigma_{4}\right)=2(2 m+n) \mu_{4} \text {. }
$$

Hence, if $\alpha_{4}$ is a generator of $H^{4}\left(S^{4}\right)$, we obtain from Theorem 3.1

$$
p_{1}\left(\mathfrak{B}_{m, n}^{(4,3)}\right)=-\bar{c}\left(\mathfrak{B}_{m, n}^{(4,3)}\right)= \pm 2(2 m+n) \alpha_{4} .
$$

For $r \geqq 5$, in the same way

$$
\left(k_{r}\right)_{*} \circ\left(j_{r}\right)_{*}\left(n \sigma_{r}\right)=\left(j_{r}^{\prime}\right)_{*} \circ(d)_{*}\left(n \sigma_{r}\right)=2 n \sigma_{2 r}=\left(k_{r}\right)_{*}\left(2 n \mu_{r}\right)
$$

therefore from Theorem 3.1

$$
p_{1}\left(\mathfrak{B}_{n}^{(4, r)}\right)=-\bar{c}\left(\mathfrak{B}_{n}^{\prime(4, r)}\right)= \pm 2 n \alpha_{4} .
$$

Thus we have

THEOREM 4.1. (i) The first Pontrjagin class $p_{1}$ of $\mathfrak{B}_{m, n}^{(4,3)}$ is $\pm 2(2 m+n) \alpha_{4}$.

(ii) For $r \geqq 4$, the first Pontrjagin class $p_{1}$ of $\mathfrak{B}_{n}^{(4, r)}$ is $\pm 2 n \alpha_{4}$, where $\alpha_{4}$ is a generator of $H^{4}\left(S^{4}\right)$.

REMARK 4.2. In the case of $\mathfrak{B}_{m, n}^{(4,3)}$, Milnor obtained the same result using Pontrjagin class of quaternion projective plane calculated by Hirzebruch (Milnor [8]). 
(II) The second Pontrjagin class of $\mathfrak{B}^{(8, r)}(r \geqq 7)$.

For $r \geqq 4$, we have $\pi_{7}(S U(r)) \approx Z$. Let $\bar{\mu}_{r}$ be a generator of $\pi_{7}(S U(r))$ such that

$$
\left(i_{r}^{\prime}\right)_{*} \bar{\mu}_{r}=\bar{\mu}_{r+1}, \quad\left(k_{5}\right)_{*} \bar{\mu}_{5}=2 \bar{\sigma}_{10} \cdot{ }^{3)}
$$

LEMMA 4.3. Let $r \geqq 7$ and $p: S U(r) \rightarrow S U(r) / S U(3)$ be natural projection, then

$$
p_{*} \bar{\mu}_{r}= \pm 6 \lambda \text {, }
$$

where $\lambda$ is a generator of $\pi_{7}(S U(r) / S U(3)) \approx Z$.

ProOF. We consider exact sequence of homotopy groups of the principal bundle $(S U(r), p, S U(r) / S U(3), S U(3))$.

$\cdots \rightarrow \pi_{7}(S U(3)) \rightarrow \pi_{7}(S U(r)) \rightarrow \pi_{7}(S U(r) / S U(3)) \rightarrow \pi_{6}(S U(3)) \rightarrow \pi_{6}(S U(r)) \rightarrow \cdots$

Now we have

$$
\begin{gathered}
\pi_{7}(S U(3))=0, \pi_{7}(S U(r)) \approx Z, \pi_{7}(S U(r) / S U(3)) \approx Z, \\
\pi_{6}(S U(3)) \approx Z_{6}, \pi_{6}(S U(r))=0
\end{gathered}
$$

whence our lemma is easily proved.

Now from the diagram (4.1) follows

$$
\begin{aligned}
\left(k_{8}\right)_{*} \circ\left(j_{8}\right)_{*}\left(m \bar{\rho}_{8}+n \bar{\sigma}_{8}\right) & =\left(j_{8}^{\prime}\right)_{* \circ}(d)_{*}\left(m \bar{\rho}_{8}+n \bar{\sigma}_{8}\right) \\
& =2\left(i_{15}\right)_{*} \circ\left(i_{14}\right)_{*} \circ \cdots \circ\left(i_{8}\right)_{*}\left(m \bar{\rho}_{8}+n \bar{\sigma}_{8}\right) .
\end{aligned}
$$

Since $\left(i_{r}\right)_{*}$ is isomorphism onto for $r \geqq 9$, and

$$
\left(i_{8}\right)_{*}\left(\bar{\rho}_{8}\right)=2 \bar{\sigma}_{9},\left(i_{8}\right)_{*} \bar{\sigma}_{8}=t \bar{\sigma}_{9},
$$

we have

$$
\left(k_{8}\right)_{*} \circ\left(j_{8}\right)_{*}\left(m \bar{\rho}_{8}+n \bar{\sigma}_{8}\right)=2(2 m+i n) \bar{\sigma}_{16} .
$$

On the other hand, we have

$$
\begin{aligned}
\bar{\mu}_{8}= & \left(i_{7}^{\prime}\right)_{*} \circ \cdots \circ\left(i_{4}^{\prime}\right)_{*} \bar{\mu}_{4}, 2 \bar{\sigma}_{16}=\left(i_{15}\right)_{*} \circ\left(i_{14}\right)_{*} \circ \cdots \circ\left(i_{8}\right)_{*} \circ\left(k_{5}\right)_{*} \bar{\mu}_{5} \\
& \left(k_{8}\right)_{*} \circ\left(i_{7}^{\prime}\right)_{*} \circ \cdots \circ\left(i_{5}^{\prime}\right)_{*}=\left(i_{15}\right)_{*} \circ\left(i_{14}\right)_{*} \circ \cdots \circ\left(i_{10}\right)_{*} \circ\left(k_{5}\right)_{*}
\end{aligned}
$$

therefore

$$
\left(j_{8}\right)_{*}\left(m \bar{\rho}_{8}+n \bar{\sigma}_{8}\right)=(2 m+t n) \bar{\mu}_{8} .
$$

For $r \geqq q$, we obtain in the same way

$$
\left(k_{r}\right)_{*} \circ\left(j_{r}\right)_{*}\left(n \bar{\sigma}_{r}\right)=\left(j_{r}^{\prime}\right)_{*} \circ(d)_{*}\left(n \bar{\sigma}_{r}\right)=2 n \bar{\sigma}_{2 r}=\left(k_{r}\right)_{*}\left(n \bar{\mu}_{r}\right) .
$$

Hence from the Lemma 4.3 and Theorem 3.1 follows 


$$
\begin{aligned}
& p_{2}\left(\mathfrak{B}_{m, n}^{(8,7)}\right)=\bar{c}\left(\mathfrak{B}_{m, n}^{\prime(8,7)}\right)= \pm 6(2 m+t n) \alpha_{8} \\
& p_{2}\left(\mathfrak{B}_{n}^{(8, r)}\right)=\bar{c}\left(\mathfrak{B}_{n}^{\prime(8, r)}\right)= \pm 6 n \alpha_{8} \quad(r \geqq 8),
\end{aligned}
$$

where $\alpha_{8}$ denotes a generator of $H^{8}\left(S^{8}\right)$.

So we obtain the following theorem:

THEOREM 4.4. (i) The second Pontrjagin class $p_{2}$ of $\mathfrak{B}_{m, n}^{(8,7)}$ is $\pm 6(2 m+t n) \alpha_{8}$, where $t$ is odd integer determined by (2.1) and $\alpha_{8}$ is a generator of $H^{8}\left(S^{8}\right)$.

(ii) For $r \geqq 8$, the second Pontrjagin class $p_{2}$ of $\mathfrak{B}_{n}^{(8, r)}$ is $\pm 6 n \alpha_{8}$.

REMARK 4.5. Pontrjagin classes defined here are different from classical ones which are defined by Grassmann manifolds. But since the difference between them is 2-torsion, both definitions coincide in our cases (Wu [16]).

REMARK 4.6. By Theorem 4.4 (i) and the fact that the homogeneous part $\Omega^{15}$ of the 15th degree of Thom algebra $\Omega$ is a finite group (Thom [13]), we obtain the manifolds which are homeomorphic, but not diffeomorphic with 15 -sphere $S^{15}$, making use of the invariant $\lambda$ constructed in the same way as Milnor [8].

\section{Cohomology groups of total spaces.}

We shall first prove the following lemmas.

LEMMA 5.1. The primary obstruction of $\mathfrak{B}_{m, n}^{(4,3)}$ is $\pm n \alpha_{4}$.

PROOF. Take quaternion unit 1 for $y_{0}$ of section 3 . Then we have $T(x)(1)=\boldsymbol{n} \sigma_{4}(1)$ by definitions of $\rho_{4}, \sigma_{4}$, and this defines $n$ multiple of a generator of $\pi_{3}\left(S^{3}\right)$. So lemma is proved by Theorem 3.1.

LEMMA 5.2. The primary obstruction of $\mathfrak{B}_{m, n}^{(8,7)}$ is $\pm n \alpha_{8}$.

PROOF. This is proved similarly as in the preceeding lemma 5.1. We may only replace $\rho_{4}, \sigma_{4}$ by $\bar{\rho}_{8}, \bar{\sigma}_{8}$, quaternion unit 1 by Cayley unit 1 .

REMARK 5.3. Obviously the primary obstruction is trivial in $\mathfrak{B}_{n}^{(4, r)}(r \geqq 4), \mathfrak{B}_{n}^{(8, r)}(r \geqq 8)$.

We now consider the Gysin exact sequence (Serre [9] Prop. 6)

$$
\cdots \rightarrow H^{i}\left(S^{q}, Z\right) \rightarrow H^{i}\left(B^{(q, r)}, Z\right) \rightarrow H^{i-r}\left(S^{q}, Z\right) \stackrel{h}{\rightarrow} H^{i+1}\left(S^{q}, Z\right) \rightarrow \cdots
$$

where $h(x)=x \cdot \bar{c}\left(\mathfrak{B}^{(q, r)}\right)$.

This enables us to compute the cohomology groups of total spaces from Lemma 4.1, 4.2. We obtain:

THEOREM 5.4. (i) Non-trivial cohomology groups of $B^{(4, r)}$ are 


$$
\begin{aligned}
& H^{4}\left(B_{m, n}^{(4,3)}\right) \approx Z_{n} \\
& H^{4}\left(B_{n}^{(4,4)}\right) \approx Z+Z \\
& H^{4}\left(B_{n}^{(4, r)}\right) \approx H^{r}\left(B_{n}^{(4, r)}\right) \approx Z \quad(r \geqq 5) .
\end{aligned}
$$

(ii) Non-trivial cohomology groups of $B^{(8, r)}$ are

$$
\begin{aligned}
& H^{8}\left(B_{m, n}^{(8, r)}\right) \approx Z_{n} \\
& H^{8}\left(B_{n}^{(8,8)}\right) \approx Z+Z \\
& H^{8}\left(B_{n}^{(8, r)}\right) \approx H^{r}\left(B_{n}^{(8, r)}\right) \approx Z \quad(r \geqq 9) .
\end{aligned}
$$

\section{Pontrjagin classes of manifolds.}

In the following sections, all manifolds and differentiable structures considered are always $C^{\infty}$-differentiable.

$S^{q}$ and $S O(r+1)$ have natural differentiable structures and $\rho_{4}, \sigma_{4}, \sigma_{r}$, $t \bar{\rho}_{8}, \bar{\sigma}_{8}, t \bar{\sigma}_{r}$ defined in section 2 are differentiable mappings.

Therefore we can define differentiable coordinate transformations in $V_{1} \cap V_{2}$ from the characteristic map $T$ (Steenrod [11] $\S 18$ ). Then open covering $V_{i} \times S^{r}(i=1,2)$ with natural differentiable structure determines a differentiable structure on $B^{(q, r)}$.

Let $M_{m, n}^{(q, r)}, M_{n}^{(q, r)}$ be manifolds thus obtained from $B_{m, n}^{(q, r)}, B_{n}^{(q, r)}$. Now, Pontrjagin classes of a manifold $M$ mean, as usual, Pontrjagin classes of its tangential bundle $\mathfrak{I}(M)$.

Let $\beta_{4}, \beta_{8}$ be the generators of $H^{4}\left(M^{(1, r)}\right), H^{8}\left(M^{(8, r)}\right)$ respectively given by $\beta_{4}=p^{*}\left(\alpha_{4}\right), \beta_{8}=p^{*}\left(\alpha_{8}\right)$, where $p$ is the projection.

THEOREM 6.1. (i) The first Pontrjagin class $p_{1}$ of $M_{m, n}^{(4,3)}$ is $\pm 4 m \beta_{4}$ $(\bmod n)$.

(ii) For $r \geqq 4$, the first Pontrjagin class $p_{2}$ of $M_{n}^{(4, r)}$ is $\pm 2 n \beta_{4}$.

THEOREM 6.2. (i) The second Pontrjagin class $p_{2}$ of $M_{t m, n}^{(8,7)}$ is $\pm 12 t m \beta_{8}(\bmod n)$.

(ii) For $r \geqq 8$, the second Pontrjagin class $p_{2}$ of $M_{t n}^{(8, r)}$ is $\pm 6 \operatorname{tn} \beta_{8}$.

PROOF. Let $\sigma^{r+1}$ be $(r+1)$-cell of closed interior of $S^{r}$. We associate to bundle $\mathfrak{B}^{(q, r)}$, the bundle $\overline{\mathfrak{B}}^{(q, r)}$ with fibre $\sigma^{r+1}$ :

$$
\overline{\mathfrak{B}}^{(q, r)}=\left\{\bar{B}^{(q, r)}, \bar{p}, S^{q}, \sigma^{r+1}, S O(r+1)\right\} \text {. }
$$

Natural differentiable structure is defined on $\bar{B}^{(q, r)}$ as $B^{(q, r)}$ above and obtain $(q+r+1)$-dimensional manifold $V^{(q, r)}$ with boundary. Obviously 
$M^{(q, r)}$ is boundary manifold of $V^{(q, r)}$.

$V^{(q, r)}$ is of the same homotopy type as $S^{q}$ and $\bar{p}^{*} \alpha_{q}$ is a generator of $H^{q}\left(V^{(q, r)}\right)$, where $\alpha_{q}$ is a generator of $H^{q}\left(S^{q}\right)$.

Let $\mathfrak{I}\left(V^{(q, r)}\right)$ be the tangential bundle of $V^{(q, r)}$. Then $\mathfrak{I}\left(V^{(q, r)}\right)$ is the Whitney sum of the bundle of vectors tangent to the fibre $\mathfrak{I}_{1}\left(V^{(q, r)}\right)$ and the bundle of vectors normal to the fibre $\mathfrak{I}_{2}\left(V^{(q, r)}\right) . \mathfrak{I}_{1}\left(V^{(q, r)}\right)$ is the induced bundle induced by $\mathfrak{B}^{(q, r)}$ and $\bar{p}$, and $\mathfrak{I}_{2}\left(V^{(q, r)}\right)$ is the one induced by tangent bundle of $S^{q}$ and $\bar{p}$. Since $S^{4}, S^{8}$ are boundary manifolds, we have $p_{1}\left(S^{\lrcorner}\right)=0, p_{2}\left(S^{8}\right)=0$. Therefore, for $q=4,8$

$$
p_{i}\left(\mathfrak{T}\left(V^{(\boldsymbol{q}, r)}\right)\right)=p_{i}\left(\mathfrak{I}_{1}\left(V^{(q, r)}\right)\right)=\bar{p}^{*} p_{i}\left(\mathfrak{B}^{(q, r)}\right) \quad(i=q / 4)
$$

Now let $i: M^{(q, r)} \rightarrow V^{(q, r)}$ be injection. Over $M^{(q, r)}, i^{*} \mathfrak{T}\left(V^{(\boldsymbol{q}, r)}\right)$ is the Whitney sum of $\mathfrak{I}\left(M^{(q, r)}\right)$ and the 1-vector bundle $\mathfrak{N}\left(M^{(q, r)}\right)$ normal to $\mathfrak{T}\left(M^{(q, r)}\right)$ in $V^{(q, r)}$. Obviously $\mathfrak{N}\left(M^{(q, r)}\right)$ is trivial, therefore

$$
p_{i}\left(\mathfrak{I}\left(M^{(q, r)}\right)\right)=i^{*} p_{i}\left(\mathfrak{I}\left(V^{(q, r)}\right)\right) \quad(i=q / 4)
$$

Since $i$ is a bundle map

$$
i^{*} \bar{p}^{*} \alpha_{q}=p^{*} \alpha_{q}=\beta_{q} .
$$

We obtain from (6.1), (6.2), (6.3)

$$
p_{i}\left(M^{(q, r)}\right)=p^{*} p_{i}\left(\mathfrak{B}^{(q, r)}\right) \quad q=4,8, i=q / 4
$$

Hence our theorems follow from Theorem 4.1, Theorem 4.4, Theorem 5.4 .

\section{Pontrjagin classes and homotopy types of manifolds.}

From Theorem 2.3, Theorem 2.4, Theorem 6.1, Theorem 6.2, we obtain

THEOREM 7.1. (i) $M_{m+12 i, n}^{(4,3)}, M_{-m+12 i^{\prime},-n}^{(4,3)}\left(i, i^{\prime}=0, \pm 1, \pm 2, \cdots\right)$ are of the same homotopy type and their first Pontrjagin classes $p_{1}$ are

$$
p_{1}\left(M_{m+12 i, n}^{(4,3)}\right)= \pm(4 m+48 i) \beta_{4} \quad \bmod n \text {. }
$$

(ii) For $r \geqq 4, M_{ \pm n+24 i}^{(4, r)}(i=0, \pm 1, \pm 2, \cdots)$ are of the same homotopy type and their first Pontrjagin classes $p_{1}$ are

$$
p_{1}\left(M_{n+24 i}^{(4, r)}\right)= \pm(2 n+48 i) \beta_{4} .
$$

THEOREM 7.2. (i) $M_{t m+\{120, t\} i, n}^{(8,7)}, M_{-t m+\{120, t\} i^{\prime},-n}^{(8,7)}\left(i, i^{\prime}=0, \pm 1, \pm 2, \cdots\right)$ are of the same homotopy type and their second Pontrjagin classes $p_{2}$ are

$$
p_{2}\left(M_{t m+\{120, t\} i, n}^{(8,7)}\right)= \pm(12 t m+12\{120, t\}) \beta_{8} \bmod n \text {. }
$$


(ii) For $r \geq 8, M_{ \pm i n+\{240, t\} i}^{(8, r)}(i=0, \pm 1, \pm 2, \cdots)$ are of the same homotopy type and their second Pontrjagin classes $p_{2}$ are

$$
p_{2}\left(M_{t n+\{240, t\} i}^{(8, r)}\right)= \pm(6 t n+6\{240, t\} i) \beta_{8} .
$$

Where $t$ is odd integer determined by (2.1) and $\{$,$\} means l.c.m.$

In particular, $M_{0,0}^{(q, r)}, M_{0}^{(q, r)}$ are $S^{q} \times S^{r}$, and so we have

COROLLARY 7.3. For $r \geq 3$, there are infinitely many $(4+r)$-dimensional manifolds of the same homotopy type as $S^{4} \times S^{r}$, whose first Pontrjagin classes $p_{1}$ are divisible by 48 , and between which there exists no diffeomorphism (i.e. differentiable homeomorphism).

COROLLARY 7.4. For $r \geqq 7$, there are infinitely many $(8+r)$-dimensional manifolds of the same homotopy type as $S^{8} \times S^{r}$, whose second Pontrjagin classes $p_{2}$ are divisible by $12\{120, t\}$, and between which there exists no diffeomorphism.

REMARK 7.5. Thom proved topological (homotopy) invariance of $p_{1}$ mod 2 (Thom [12]). Wu and Hirzebruch proved topological (homotopy) invariance of $p_{1} \bmod 3(\mathrm{Wu}$ [15], [16], Hirzebruch [3]).

The results obtained above would disprove the topological invariance of Pontrjagin classes, if the conjecture of Hurewicz "Two closed manifolds of the same homotopy type is homeomorphic" (Hurewicz [6]) is true, or if, what would be easier to prove, one of $M_{ \pm 12 m, 0}(m=$ $1,2, \cdots)$ is homeomorphic to $S^{4} \times S^{3}$ (Hirzebruch [4] Problem 1).

Conversely if topological invariance of $p_{1}$ for $\bmod p, p$ being a prime other than 2,3, or topological invariance of $p_{2}$ for $\bmod p, p$ being a prime other than $2,3,5$ and factors of $t$, could be proved, then the conjecture of Hurewicz would be denied.

University of Tokyo

\section{Notes}

1) The author does not know whether $t=1$ or not, and also whether $\bar{\rho}_{7}$ has $C^{\infty}$. differentiable representative or not.

2) Notice that the kernel of $\left(i_{8}\right)_{*}$ is generated by $2 \bar{\sigma}_{8}-t \bar{\rho}_{8}$.

3) The coefficient of $\bar{\sigma}_{10}$ must be \pm 1 or \pm 2 by (4.2). On the other hand, we can show that it is even, by using relations (2.2) and the exact sequence of homotopy groups of the principal bundle ( $\left.S U(4), S^{7}, S U(3)\right)$. 


\section{Bibliography}

[1] A. Borel and J.-P. Serre, Groupes de Lie et Puissances Réduites de Steenrod, Amer. J. Math., 75 (1953), pp. 409-448.

[2] A. Dold, Über Fasernweise Homotopieäquivalenz von Faserräumen, Math. Zeit., 62 (1955), pp. 111-136.

[3] F. Hirzebruch, On Steenrod Reduced Powers in Oriented Manifolds, Notes Princeton University (1953), mimeographed.

[4] F. Hirzebruch, Some Problems on Differentiable and Complex Manifolds, Ann. of Math., 60 (1954), pp. 213-236.

[5] F. Hirzebruch, Neue Topologische Methoden in der Algebraischen Geometrie, (1956).

[6] W. Hurewicz, Beiträge zur Topologie der Deformationen III, Proc. Acad. Amsterdam, 39 (1936), pp. 117-126.

[7] I. M. James and J. H. C. Whitehead, The Homotopy Theory of Sphere Bundles over Spheres, Proc. London Math. Soc., 4 (1954), pp. 196-218.

[8] J. Milnor, On Manifolds Homeomorphic to the 7-Sphere, Ann. of Math., 64 (1956), pp. 339-405.

[9] J.-P. Serre, Homologie Singulière des Espaces Fibrés, Ann. of Math., 54 (1951), pp. $425-505$.

[10] J.-P. Serre, Quelques Calculs de Groupes d'Homotopie, C. R. Acad. Sci. Paris, 236 (1953), pp. $2475-2477$.

[11] N. Steenrod, The Topology of Fibre Bundles. (1951).

[12] R. Thom, Espaces Fibrés en Sphères et Carrés de Steenrod, Ann. Sci. Ecole Norm. Sup., 69 (1952).

[13] R. Thom, Quelques propriétés globales des variétés différentiables, Comment. Math. Helv., 28 (1954), pp. 17-86.

[14] G. W. Whitehead, A generalization of the Hopf invariant, Ann. of Math., 51 (1950), pp. 192-237.

[15] W. T. Wu, Sur les Puissances de Steenrod (Colloque de Topologie de Strasbourg) (1951).

[16] W. T. Wu, On Pontrjagin Classes I, II, Scientia Sinica III (1954), pp. 353-367, IV (1955), pp. 455-490. 